\title{
Le sujet en position d'enseignant : pratiques et discours de trois professeurs d'EPS débutants avec ou sans expérience personnelle dans l'activité enseignée
}

The subject as a teacher: practices and speeches of three novice physical education teachers with or without personal experience in the taught activity

\section{Pablo Buznic-Bourgeacq et André Terrisse}

\section{(2)enEdition} Journals

\section{Édition électronique}

URL : http://journals.openedition.org/rfp/4209

DOI : 10.4000/rfp.4209

ISSN : 2105-2913

\section{Éditeur}

ENS Éditions

\section{Édition imprimée}

Date de publication : 15 décembre 2013

Pagination : 15-28

ISBN : 978-2-84788-521-7

ISSN : 0556-7807

\section{Référence électronique}

Pablo Buznic-Bourgeacq et André Terrisse, « Le sujet en position d'enseignant : pratiques et discours de trois professeurs d'EPS débutants avec ou sans expérience personnelle dans l'activité enseignée », Revue française de pédagogie [En ligne], 184 | 2013, mis en ligne le 15 décembre 2016, consulté le 30 avril 2019. URL : http://journals.openedition.org/rfp/4209; DOI : 10.4000/rfp.4209 


\section{Le sujet en position d'enseignant : pratiques et discours de trois professeurs d'EPS débutants avec ou sans expérience personnelle dans l'activité enseignée}

Pablo Buznic-Bourgeacq et André Terrisse

Enseigner suppose de savoir. Ce texte questionne l'écart entre cette supposition et les pratiques effectives des enseignants débutants. Nous y analysons les pratiques et les discours de trois professeurs d'éducation physique et sportive (EPS) débutants, enseignant des activités physiques sportives et artistiques (APSA) dans lesquelles ils ont une expérience personnelle soit prolongée soit inexistante. À partir d'une approche didactique clinique des pratiques d'enseignement, nous décrivons leur activité didactique et leur positionnement subjectif en classe. Nous montrons alors comment la position d'enseignant, qui définit chaque débutant sans être encore la sienne, constitue une construction subjective, enchevêtrée d'assujettissements, d'enjeux narcissiques et de manque, qui singularise la question du poids de l'expérience chez chaque enseignant débutant.

Mots-clés (TESE) : enseignant, didactique, éducation physique, expérience professionnelle.

\section{L'EXPÉRIENCE PERSONNELLE DU SUJET ENSEIGNANT}

\section{D'une situation professionnelle critique}

S'il ne suffit pas de savoir pour enseigner, la figure du " maître instruit " (Paquay, 1994) s'est instaurée comme définition initiale de la professionnalité enseignante (Shulman, 1986 ; Altet, 1996). L'enseignant est celui qui sait, celui qui maîtrise ce qu'il va enseigner.

Plusieurs recherches interrogeant les pratiques et les discours des acteurs de l'enseignement (BuznicBourgeacq, Terrisse \& Margnes, 2007 ; BuznicBourgeacq, Terrisse \& Lestel, 2008 ; Philippot, 2008) s'entendent à montrer que la question de la maîtrise des savoirs disciplinaires demeure centrale dans les pratiques quotidiennes. Certains degrés et disciplines d'enseignement sous-tendent une maîtrise particulièrement vaste et diversifiée de savoirs, peu envisageable de manière exhaustive et homogène (Carnus, 2010). Les professeurs d'éducation physique et sportive (EPS) enseignent une multiplicité d'activités physiques sportives et artistiques (APSA), de la danse au rugby, sans avoir pu eux-mêmes toutes les pratiquer (Buznic-Bourgeacq, 2009 ; Ben Jomaa, 2011 ; Touboul, Carnus \& Terisse, 2012).

Ce texte, en s'appuyant sur nos travaux en didactique clinique de l'EPS (Buznic-Bourgeacq, Terrisse \& Margnes, 2007, 2010 ; Buznic-Bourgeacq, Terrisse 
\& Lestel, 2008), questionne cette situation professionnelle à partir de la problématique de l'expérience personnelle du sujet enseignant.

\section{Une problématique de recherche en didactique de I'EPS}

" C'est ça la difficulté du métier de toute façon... c'est devoir enseigner une activité que tu connais pas... " Ainsi concluait l'enseignante au cœur d'une première série de recherches sur cette problématique (Buznic-Bourgeacq, Terrisse \& Margnes, 2007).

Pour aborder la question de la maîtrise des savoirs disciplinaires, nos travaux tentent d'identifier l'influence de l'expérience personnelle du professeur d'EPS dans les APSA qu'il a à charge d'enseigner sur ses pratiques effectives d'enseignement. Nous nous interrogeons sur le savoir construit dans l'expérience personnelle de pratiquant et sur sa transmission en classe par un sujet devenu enseignant. La problématique est donc en premier lieu didactique. II s'agit d'identifier les processus transpositifs qui viennent réorganiser les savoirs, de l'expérience personnelle du sujet à l'enseignement effectif du sujet enseignant (Arnaud, 1986 ; Terrisse, 2001 ; Buznic-Bourgeacq, 2009).

\section{Une problématique de recherche en didactique clinique}

Notre intérêt pour cette problématique est orienté par notre ancrage en didactique clinique (Terrisse \& Carnus, 2009 ; Carnus \& Terrisse, 2013), par la posture épistémologique, théorique et méthodologique qu'il sous-tend : la prise en compte du sujet dans la recherche (Terrisse, 2000). Le sujet et la subjectivité constituent le cœur des épistémologies, théories et méthodologies d'une approche clinique en sciences humaines (Revault d'Allonnes, 1989). D'un point de vue épistémologique, la construction du savoir scientifique en est revisitée : au filtre du sujet, ce savoir est considéré dans sa singularité, comme co-construit par le sujet chercheur et le sujet de la recherche, tous deux traversés par des processus psychiques inconscients. D'un point de vue méthodologique, les études de cas s'imposent, et le discours du sujet y trouve une place fondamentale. En ce qui concerne la théorie, la condition est bien celle de la référence à une théorie du sujet pour décrire et interpréter les phénomènes.

La problématique de l'expérience interroge les pratiques enseignantes à partir d'un point critique : les rapports entre l'enseignant et le savoir, dans ce qu'ils spécifient la singularité de sa position subjective dans le didactique (Buznic-Bourgeacq, 2009). Cette problématique constitue en effet une loupe sur le sujet enseignant, considérant qu'une expérience prolongée de pratique dans une activité ou au contraire une absence totale d'expérience personnelle exacerbe le rôle et le statut du savoir pour le sujet.

Notre travail s'inscrit ainsi dans un projet scientifique (Terrisse, 1994 ; Carnus \& Terrisse, 2013) consistant à identifier dans l'activité didactique de l'enseignant ce qui l'organise en tant que sujet. Car cette activité en classe n'est pas uniquement organisée par des contraintes didactiques et institutionnelles, mais aussi par des contraintes internes (Blanchard-Laville, 2001). L'enseignant est traversé par des enjeux subjectifs (Buznic-Bourgeacq, Terrisse \& Margnes, 2010) qui organisent son activité didactique effective, c'est-àdire l'activité qu'il déploie de manière usuelle dans sa classe à propos d'objets de savoir qu'il a à charge de faire reconstruire par ses élèves. Ce travail montre ainsi comment la prise en compte conjointe de ces enjeux et de l'activité didactique en classe peut permettre de proposer un regard compréhensif et heuristique sur les processus didactiques.

\section{PERSPECTIVES EN DIDACTIQUE CLINIQUE : ANALYSE DES PRATIQUES ET THÉORIE DU SUJET DIDACTIQUE}

\section{Analyses des pratiques enseignantes : des outils pour décrire le didactique}

Dans le champ de l'analyse des pratiques d'enseignement, nos cadres descriptifs et interprétatifs se situent au croisement des approches didactique et clinique. D'un point de vue didactique, nous nous référons à trois ensembles conceptuels pour décrire le champ de la transmission des savoirs : la théorie des situations didactiques de G. Brousseau (1998), les théories de l'action didactique élaborées à partir de l'émergence de la didactique comparée (Mercier, Schubauer-Leoni \& Sensevy, 2002) et les travaux de G. Vergnaud (2002) sur le concept de schème1.

Pour cette recherche, nous avons construit un ensemble d'indicateurs empiriques articulés entre eux et témoignant des procédures didactiques mises en œuvre par les enseignants ${ }^{2}$. Nous nous attachons à décrire structurellement les savoirs mis en jeu à partir des composants du schème définis par 
G. Vergnaud (1990) - invariants, buts, règles, inférences, signifiants - de leurs relations, leur diversité, leur redondance au fil des interactions. Ce regard épistémologique permet de dégager des premiers éléments significatifs de logiques didactiques singulières chez les enseignants, centrées sur les formes prévalentes des savoirs enseignés : des automatismes, des principes logiques, des pratiques, etc. À partir de ces éléments, nous décrivons alors l'activité interactionnelle des enseignants avec les élèves à propos des savoirs. Les concepts de milieu et de contrat didactique (Brousseau, 1998) nous permettent d'identifier le jeu didactique instauré et de décrire l'activité didactique de l'enseignant. Nous nous centrons ici sur ses dimensions topogénétique, relative au " partage des tâches et des responsabilités de chacun vis-à-vis du savoir enseigné ", et mesogenétique, relative à " l'aménagement progressif d'un milieu » (Loquet, Garnier \& Amade-Escot, 2002).

L'analyse des pratiques se poursuit donc en décrivant l'activité didactique de l'enseignant sur et dans le milieu de l'élève, la manière dont il se situe par rapport à ce milieu, l'intensité avec laquelle il l'aménage et fixe l'élève dans un contrat didactique spécifique. Elle conduit à identifier comment l'enseignant donne et reprend les responsabilités de l'émergence du savoir, au travers du milieu de l'élève et par des procédures ostensives (Salin, 2002). Ces cadres d'analyse nous permettent alors de décrire les pratiques de chaque enseignant et d'en dégager leur logique didactique singulière.

L'intention de reconstruire cette singularité nous amène à ouvrir nos cadres au sujet enseignant. D'un point de vue clinique, sa prise en compte passe donc par la référence à une théorie du sujet (Terrisse, Carnus \& Sauvegrain, 2002 ; Terrisse, 2008). Nous nous référons ici à la psychanalyse lacanienne (Lacan, 1974).

\section{Le " sujet didactique " : une théorie du sujet en didactique}

Au travers d'études de cas, nous avons pu identifier combien l'activité didactique singulière de chaque enseignant est traversée par des enjeux subjectifs liés à l'assomption d'une image et d'une fonction symbolique toujours en construction ; combien aussi cette activité se répète, face à des impossibles (Terrisse, 2009) pour le sujet. Nous avons alors organisé ces éléments, en confrontant nos études de cas, au travers d'" une théorie du sujet, sous la forme [...] d'une structure (RSI), susceptible de rendre compte de la division d'un sujet confronté à plusieurs déterminations... et à l'épreuve de la classe » (Terrisse, 2008). La référence aux registres lacaniens - Réel, Symbolique, Imaginaire (Lacan, 1974) - pour envisager les coordonnées du sujet émerge donc de son heuristique épistémologique pour envisager les modes d'organisation d'un sujet en position d'enseignant.

Le symbolique et la fonction de "sujet supposé savoir ": l'enseignant est d'abord fonction de la transmission des savoirs et, plus spécifiquement, fonction de l'institution scolaire. Représentant institutionnel du savoir, il se retrouve d'emblée assigné à une place d'où il est défini. Son mode d'existence renvoie ici à une inscription dans un ordre symbolique qui, de le précéder, le détermine fondamentalement. Le registre lacanien du symbolique est bien celui de l'assujettissement, du langage, de la loi, de « l'ensemble des institutions qui précèdent la naissance du sujet et le prédéterminent " (Assoun, 2003, p. 50). Dans cette perspective, le sujet didactique est donc fonction d'une place, inscrite dans un ordre symbolique " disposé selon une chaîne signifiante autonome, extérieure au sujet [...] au regard duquel ce sujet ne peut qu'exister sur un mode acéphale, c'està-dire tout entier assujetti à cet ordre » (Chemama, 2009, p. 562). Enseignant, maître, éducateur, professionnel, " il n'a pas de "rôle" à apprendre et pourtant il s'y retrouve piégé » (Pujade-Renaud, 1983, p. 76).

Pour autant, sa position institutionnelle par rapport au savoir lui attribue la responsabilité de sa transmission et le place de fait à une position symbolique supposée, celle de "sujet supposé savoir ". Ce syntagme élaboré par J. Lacan (1966) pour caractériser la position symbolique de l'analyste a été repris, de manière ponctuelle (Chevallard, 1991 ; Terrisse, 1994 ; Blanchard-Laville, 2001 ; Buznic-Bourgeacq, 2009, 2013), pour souligner la position toujours supposée qui situe l'enseignant. Le sujet didactique est supposé savoir pour l'élève. Nous avons pu alors identifier dans nos travaux combien cette position symbolique, cette supposition permanente intériorisée, organise l'activité didactique des enseignants débutants ; chacun cherchant à l'incarner ou à la fuir singulièrement, naviguant dans l'écart avec lui-même ouvert par l'assujettissement.

L'imaginaire et la forme reflétée du « maître »: la relation duale qui unit l'enseignant à l'élève dans la classe le confronte à " une dimension de l'autre où l'altérité d'une certaine façon s'efface, les partenaires tendant à se ressembler de plus en plus " (Chemama, 2009 , p. 79). Cette relation faite d'identifications - « transformation(s) produite(s) chez le sujet quand il assume une image " (Lacan, 1966, p. 84) - s'inscrit 
dans le registre de l'imaginaire, instance de " la confusion entre soi et l'autre " (Chemama, 2009, p. 352). Le sujet ${ }^{3} s^{\prime} y$ définit dans sa relation à l'autre, dans laquelle "quelque chose de factice s'introduit toujours qui est la projection de l'un sur le simple écran que devient l'autre " (Chemama, 2009, p. 267). Cet élément factice, c'est son image, qui le «forme " et avec laquelle il se confond. Son assomption, sous la forme d'allers-retours entre image perçue et image idéalisée, constitue le support du narcissisme.

Les pratiques d'enseignement sont propices à être analysées au filtre de cette dimension (PujadeRenaud, 1983 ; Filloux, 1996 ; Blanchard-Laville, 2001). En effet, confronté aux élèves, l'enseignant se retrouve face à lui-même en tant que pratiquant de l'activité enseignée, mais aussi en tant qu'apprenant, élève, enfant. D'autre part, " être enseignant, c'est être regardé " (Pujade-Renaud, 1983, p. 27). Dans la classe, l'enseignant se donne à voir. Dès lors, il assume une image qui le constitue. Or cette image, de son articulation avec la fonction de " sujet supposé savoir ", se retrouve elle aussi attendue. "L'enseignant est assigné à conserver cette place de premier, une place de maîtrise dans cette relation "inégalitaire" au savoir " (Terrisse, 1999). Le sujet didactique, s'il est fonction d'une place, s'instaure aussi comme image de maîtrise, une forme reflétée par/sur l'élève du maître. Pour le sujet didactique, " toute puissance narcissique et figure légale d'autorité s'articulent »(Pujade-Renaud, 1983, p. 78). Nous avons pu alors identifier, chez des enseignants débutants, comment l'assomption, délicate ou glorieuse, d'une image s'inscrit dans une affirmation narcissique autant qu'en soutien ou en décalage d'une position symbolique supposée. Le registre imaginaire peut agir soit de manière autonome, les enjeux narcissiques s'actualisant en classe dans des effets de prestance, de séduction ou de rivalité, soit dans son articulation avec le registre symbolique, lorsque l'image de maîtrise vient servir de légitimation au crédit initial donné au « sujet supposé savoir ».

Le réel et l'impossible du sujet : formé par une image reflétée et fonction d'une place, le sujet didactique reste pour l'instant défini à partir d'une certaine incomplétude, d'un " manque à être, dans la mesure où il n'est jamais que représenté, sup-posé (subjectum) "(Chemama, 2009, p. 99). Demeure alors un espace pour le repérer, référable au registre du réel qui " dans la réalité du sujet désigne un point qui échappe ou résiste à la symbolisation » (Lacan, 1974). Le sujet est toujours plus qu'un assujettissement. En conférant un cadre symbolique à ses actions et perceptions, il « repousse hors de ce champ un réel que, dès lors, il met en place et qui, pour lui, reste toujours présent»(Chemama, 2009, p. 493). Ainsi, " le réel est ici ce qui revient toujours à la même place " (Lacan, 2001, p. 160). De ne pas s'être inscrit dans un ordre symbolique, "il se notifie comme répétition insistante » (Assoun, 2003, p. 56).

Concernant l'enseignant, c'est son rapport à son propre assujettissement qui est ici considéré. Car, à être défini par sa position symbolique, le sujet « subit une aliénation, celle d'occuper un rang dans une suite ", réduit à " n'être que l'un des termes d'une série » (Terrisse \& Labridy, 1990), et il a à le supporter à sa manière. Le sujet didactique a été et demeure sujet, une part non assujettie à sa position symbolique dans le didactique. II s'instaure alors d'emblée en dialectique où le "sujet supposé savoir " peut buter sur un point de réel et se confronter à un impossible à supporter (Terrisse, 2009), prenant sa source dans une expérience personnelle singulière. Des expériences subjectives d'“ élève en difficulté ", de " grand frère " ou de " danseur professionnel " vont ainsi baliser les possibilités pour l'enseignant de supporter sa position symbolique dans l'espace didactique. Nous avons pu identifier, chez des enseignants, combien cette part du sujet organisait son activité en classe autour d'impossibles singuliers, allant du " charabia de la pratique sans théorie " qui conduira Nils à proposer des formalisations théoriques du rugby et de la motricité relativement pointues à ses élèves de 6 e (Buznic-Bourgeacq, 2009) aux « gens qui n'ont pas la soif d'apprendre " qui conduiront Anita à abandonner l'enseignement scolaire (Touboul, Carnus \& Terisse, 2012).

Ces axes d'analyse du sujet didactique ont été présentés selon leur genèse progressive dans nos travaux au fil des cas d'enseignants étudiés. Leur systématisation après-coup nous amène alors à proposer une structure permettant de prendre en compte chaque sujet enseignant, en l'y situant dans sa singularité.

\section{MÉTHODOLOGIE : PRATIQUES ET DISCOURS DE TROIS SUJETS ENSEIGNANTS}

\section{Terrain de la recherche}

La recherche s'organise autour des cas de trois professeurs d'EPS débutants, lors de leur première année d'enseignement au collège. Notre analyse a porté sur les enseignements menés par chaque 


\begin{tabular}{|l|c|c|c|c|}
\cline { 2 - 5 } \multicolumn{1}{c|}{} & Basket & Rugby & Natation & Danse \\
\hline Charles & NEx & & Ex & NEx \\
\hline Sam & NEx & Ex & & Ex \\
\hline Tom & NEx & & & \\
\hline
\end{tabular}

Note : Ex : Expérience prolongée dans l'APSA ; NEx : Aucune expérience dans l'APSA

enseignant dans deux ou trois APSA (tableau 1), dans lesquelles il détient une expérience personnelle de pratique prolongée (plus de cinq ans de pratique extrascolaire) ou inexistante (aucune pratique extrascolaire).

Les croisements intra et intersubjectifs, intra et inter-APSA, avec ou sans expérience, nous ont permis d'identifier progressivement le jeu d'influences qui s'instaure entre l'expérience personnelle des enseignants et leurs pratiques effectives, ainsi que la logique singulière qui organise les pratiques de chacun.

\section{Recueil des données : pratiques et discours}

Pour chacun des sept enseignements analysés, trois séances consécutives (séances 2, 3 et 4) ont été entièrement filmées 4 . Les interactions verbales ont été retranscrites dans leur intégralité. Pour assurer la compréhension des interactions didactiques, une partie de l'activité non verbale de l'enseignant et des élèves a été décrite : les procédures ostensives (Salin, 2002) non verbales de l'enseignant (démonstrations, manipulations, schématisations) ${ }^{5}$, les modifications du milieu matériel, l'activité et les performances des élèves à partir desquelles l'enseignant intervient.
Nous avons ensuite mené, puis retranscrit intégralement, plusieurs entretiens. Chaque séance observée a été suivie d'un entretien interrogeant l'enseignant sur ses intentions didactiques et leurs liens avec l'activité didactique déployée en classe, sur tout ce qui était advenu sans avoir été prévu et sur ce qui l'avait le plus préoccupé. Enfin, plusieurs mois après les observations, nous avons conduit un entretien d'“ après-coup » (Terrisse, 2007) avec chaque enseignant 6 . Nous avons alors pu identifier leurs propres descriptions et interprétations des processus en jeu dans l'accomplissement de leur nouveau métier.

\section{Exploitation des données : la construction des cas}

L'ensemble des données recueillies a été analysé progressivement. Quatre étapes ont constitué la démarche d'exploitation (figure 1).

Ces quatre étapes nous ont permis d'intégrer progressivement la spécificité de chaque APSA et la singularité de chaque enseignant dans l'identification du poids de l'expérience personnelle sur les pratiques d'enseignement. Après avoir procédé au découpage didactique des séances et des situations, l'analyse quantitative a conduit à comparer le contenu et la forme

Figure 1. Démarche d'exploitation des données

\begin{tabular}{|c|c|c|c|}
\hline $\begin{array}{c}\text { 1. “ Découpage } \\
\text { didactique ": axes } \\
\text { de lecture des } \\
\text { situations didactiques } \\
\text { (Brousseau, 1998) : } \\
\text { enchaînement des } \\
\text { situations didactiques } \\
\text { et des enjeux de savoir }\end{array}$ & $\begin{array}{l}\text { 2. Comparaisons } \\
\text { macroscopiques des } \\
\text { pratiques avec et } \\
\text { sans expérience : } \\
\text { à partir des } \\
\text { indicateurs empiriques } \\
\text { quantitatifs: } \\
\text { les tendances } \\
\text { génériques du poids } \\
\text { de l'expérience }\end{array}$ & $\begin{array}{l}\text { 3. Analyse qualitative } \\
\text { des pratiques et } \\
\text { discours de chaque } \\
\text { enseignant : analyse } \\
\text { qualitative des } \\
\text { résultats quantitatifs } \\
\text { significatifs pour } \\
\text { chacun : identification } \\
\text { de logiques didactiques } \\
\text { singulières } \\
\text { Construction } \\
\text { des cas (1) }\end{array}$ & $\begin{array}{l}\text { 4. Analyse clinique } \\
\text { des discours de } \\
\text { chaque enseignant : } \\
\text { au regard des logiques } \\
\text { didactiques singulières } \\
\text { identifiées, analyse } \\
\text { des enjeux subjectifs : } \\
\text { le positionnement } \\
\text { subjectif de chaque } \\
\text { enseignant } \\
\text { Construction } \\
\text { des cas (2) }\end{array}$ \\
\hline
\end{tabular}


des différents enseignements Ex et NEx. Les indicateurs empiriques relatifs à cette démarche se réfèrent à une actualisation des théories didactiques présentées plus haut. Au sein d'un cadre systémique d'analyse (Buznic-Bourgeacq, 2009), nous avons pu identifier et quantifier dans la totalité des interventions didactiques de chaque enseignant : la diversité et la redondance des invariants mis en jeu et leurs différentes formes d'articulations, la diversité et l'appartenance des signifiants utilisés, la multiplicité des sensations et des repères sensoriels proposés, les formes d'articulation entre les différentes situations, la fréquence du discours analogique, la potentialité des choix offerts aux élèves pour faire émerger du savoir, ou encore le degré de complexité conservé au sein de chaque situation d'apprentissage. La quantification descriptive du contenu et de la forme de ces interventions a permis d'identifier des tendances génériques dans les enseignements Ex et NEx. Elle a aussi permis de dégager des configurations spécifiques à chaque enseignant. En nous arrêtant sur les résultats les plus significatifs pour chacun (valeurs minimales et maximales relatives à différents indicateurs empiriques), nous avons décloisonné le cadre d'analyse initial pour faire émerger les problématiques propres à chaque cas. Ces problématiques spécifiques, identifiées comme logiques didactiques singulières, ont alors constitué la structure de l'analyse thématique de contenu que nous avons menée sur la retranscription des entretiens conduits avec les enseignants. Nous nous sommes attachés à identifier dans le discours interprétatif de leurs propres pratiques les enjeux subjectifs sous-jacents à chaque logique didactique. Nous avons alors analysé ces enjeux au travers du cadre théorique du "sujet didactique » présenté plus haut, en cherchant à comprendre leur mode de positionnement subjectif dans l'espace didactique.

En nous appuyant directement sur les tendances génériques dégagées de l'analyse quantitative, nous présentons, dans le cadre de cet article, la construction progressive de chaque cas.

\section{ANALYSE DES PRATIQUES : QUATRE LOGIQUES DIDACTIQUES SINGULIĖRES}

\section{Les tendances génériques les plus significatives}

Afin de pouvoir situer chaque cas, nous présentons de manière synthétique les tendances génériques qui organisent chaque ensemble d'enseignements (Ex ou $\mathrm{NEx}$ ) et révèlent de profondes spécificités.
D'une part, le contenu des enseignements Ex est beaucoup plus ouvert, dynamique et complexe. Les enseignants prennent davantage le temps de formuler ce contenu, de le rendre signifiant ${ }^{7}$, de l'extraire de la pratique de l'élève, de le référer à des éléments passés, futurs ou extérieurs à chaque situation, au travers de processus inférentiels ${ }^{8}$. Ce contenu articule davantage d'objets invariants distincts ${ }^{9}$ dans des espaces-temps distincts. Ils appuient leurs enseignements sur des situations moins didactisées, plus proches de la complexité effective de la pratique globale de I'APSA ${ }^{10}$. Leur contenu est ainsi moins bloqué dans les aménagements et le contrat didactique (Buznic-Bourgeacq, Terrisse \& Margnes, 2010).

D'autre part, dans les enseignements Ex, les enseignants se situent de manière très spécifique par rapport au milieu de l'élève. Ils s'y engagent largement plus avec leur corps ${ }^{11}$. Celui-ci est beaucoup plus source d'émergence du savoir, parfois même uniquement le modèle à suivre. Ils sont beaucoup plus proches des élèves, et l'émergence du contenu s'avère alors relative à la singularité de chaque situation objective12 (Brousseau, 1998). Faisant largement référence à leur corps performant, ils ont tendance à être plus exigeants vis-à-vis de la motricité des élèves, et les formes corporelles constituent des objets d'enseignement à part entière (BuznicBourgeacq, Terrisse \& Margnes, 2010).

\section{Trois cas : logique didactique et poids de l'expérience}

La description relative à chaque enseignant s'appuie sur plusieurs échelles de données : des éléments quantitatifs renvoyant aux tendances propres à chaque enseignant et des extraits du discours de l'enseignant en classe faisant référence à ces tendances ou à des épisodes significatifs (Loquet, Garnier \& Amade-Escot, 2002).

La logique didactique singulière qui spécifie les pratiques de chaque enseignant constitue une " configuration problématique ", un " agencement " (Passeron \& Revel, 2005) qui signe (BlanchardLaville, 2001) la singularité de chaque cas. Fondée sur une méthodologie de " construction de cas " (Terrisse, Buznic-Bourgeacq, 2011), la caractérisation de cette logique passe par la reconnaissance d'une cohérence interne dans l'activité et la parole du sujet. 
Tableau 2. Logique didactique et poids spécifique de l'expérience chez Charles

\begin{tabular}{|l|l|}
\hline \multicolumn{2}{|c|}{ Charles : natation (Ex), basket (NEx) et danse (NEx) } \\
\hline Logique didactique & Poids spécifique de l'expérience \\
\hline Enseigner? & Ex \\
Une gestion intensive du contrat didactique & Les allures de l'inexpérience \\
\cline { 2 - 2 } Des savoirs? & NEx \\
Une pratique dans un milieu spécifique & Une didactisation tenace et l'absence du corps \\
\hline
\end{tabular}

Charles : "Gérer »

Charles est dans une logique de gestion du contrat didactique (voir tableau 2). II s'attache à définir longuement l'organisation didactique des situations et régule l'activité des élèves en revenant en permanence sur cette organisation. Par exemple, lorsqu'en danse un élève vient lui soumettre sa difficulté à construire une chorégraphie, Charles lui répond simplement : « et bien oui pourquoi... parce que vous n'êtes pas dans la situation! » (D2)13. II intervient peu sur le contenu lui-même, ne l'explicite et ne le démontre presque jamais et n'enseigne qu'au travers d'aménagements didactiques relativement conséquents. II enseigne donc des pratiques au sein de milieux spécifiquement aménagés, sans véritablement en extraire des savoirs.

L'activité didactique de Charles dans ses différents enseignements est relativement semblable. En référence aux tendances génériques, les enseignements de Charles prennent tous la forme des enseignements NEx ${ }^{14}$. Son enseignement de natation (Ex) prend alors ici les allures de l'inexpérience.

Toutefois, en danse et en basket, ses enseignements se spécifient par la prégnance d'une didactisation tenace, c'est-à-dire la mise en place et le maintien persistant d'un aménagement intensif du milieu de l'élève, et une absence du corps de l'enseignant. D'une part, Charles y propose des situations conséquemment aménagées aux élèves tout en explicitant le lien entre les contraintes didactiques et les enjeux de savoir. II explicite par exemple le lien entre la contrainte " imiter les animaux » et l'enjeu de savoir qui le justifie (" utiliser différents niveaux de l'espace de danse ") : " on a pris les animaux pour qu'il y ait du sol, du marcher, vers le haut, pour que ce soit riche à ce niveau-là »(D1). Charles s'attache alors davantage à l'engagement des élèves dans le contrat didactique qu'à l'émergence potentielle du savoir. Lorsqu'en basket le contrat impose aux élèves de réaliser des " tirs en course " pour tirer au panier et que les élèves ne le respectent pas, Charles préfère ne pas lâcher le contrat, même si celui-ci conduit à l'échec des élèves : " je veux voir des tirs en course, même s'il n'y a pas de réussite " (B2). II ne négocie alors pas les éventuels contournements des élèves parfois pris de confusion qui viennent lui demander conseil : " je veux que tu fasses une situation, je veux pas t'entendre! » (B3). D'autre part, Charles ne s'engage en aucun cas corporellement dans le milieu des élèves et se limite à leurs propres productions : " ok, vous voulez une démonstration... qui c'est qui sait me le faire ? » (B3).

Sam : "Répéter »

Sam est dans une logique de répétition et d'automatisation des conduites des élèves (voir tableau 3). Le contenu de ses enseignements renvoie en grande partie à un ensemble de règles d'action, de type

Tableau 3. Logique didactique et poids spécifique de l'expérience chez Sam

\begin{tabular}{|l|l|}
\hline \multicolumn{2}{|c|}{ Sam : rugby (Ex) et basket (NEx) } \\
\hline Logique didactique & Poids spécifique de l'expérience \\
\hline \multirow{2}{*}{$\begin{array}{l}\text { Enseigner? } \\
\text { Une répétition progressive } \\
\text { Des savoirs? } \\
\text { Des automatismes }\end{array}$} & $\begin{array}{l}\text { Ex } \\
\text { L'excellence corporelle de l'enseignant }\end{array}$ \\
\cline { 2 - 2 } & NEx \\
& Des injonctions de l'extérieur \\
\hline
\end{tabular}


Tableau 4. Logique didactique et poids spécifique de l'expérience chez Tom

\begin{tabular}{|c|c|}
\hline \multicolumn{2}{|c|}{ Tom : danse (Ex) et basket (NEx) } \\
\hline Logique didactique & Poids spécifique de l'expérience \\
\hline \multirow{2}{*}{$\begin{array}{l}\text { Enseigner? } \\
\text { Une immersion globale } \\
\text { Des savoirs? } \\
\text { Une culture }\end{array}$} & $\begin{array}{l}\text { Ex } \\
\text { Une immersion à l'école de danse }\end{array}$ \\
\hline & $\begin{array}{l}\text { NEx } \\
\text { Des commentaires pour enseigner }\end{array}$ \\
\hline
\end{tabular}

« si... condition... alors... action »15. Dans cette logique, les interventions de Sam consistent principalement en des constats et des injonctions en situation ; d'une forme très directive, elles ont presque toujours pour objet l'explicitation de ce qu'il faut faire ici et maintenant : " retourne-toi », "va l'aider », « soutiens », « plaque-le» (R1).

Si les enseignements observés peuvent apparaître semblables, en rugby, Sam fait de son propre corps le premier média de la transmission du savoir ${ }^{16}$. Mettant en place des situations de jeu très proches de la pratique globale de l'activité, Sam enseigne tout en pratiquant avec les élèves. II endosse alors un double rôle : celui de capitaine d'équipe et celui de professeur d'EPS. Intervenant sans cesse dans le milieu des élèves, il régule leur activité en faisant référence à la qualité des actions corporelles qu'il met lui-même en œuvre. Lorsqu'il demande aux élèves de s'écarter pour produire un jeu déployé, il réalise lui-même l'injonction : "écarte-toi au fond" (Sam s'écarte). Lorsqu'il leur demande de venir en soutien proximal de leur coéquipier porteur de balle, il en fait autant : " on va l'aider » (Sam va aider un coéquipier) (R1). Sam alterne donc le jeu avec les élèves, dans une démonstration et une explicitation permanente de son excellence corporelle, et l'arrêt du jeu pour revenir sur ses propres actions au milieu des élèves et montrer corporellement ce qu'il faut faire. II insiste par exemple sur l'impossibilité pour les élèves de le mettre au sol : " je tomberai pas, vous arriverez pas à me plaquer » (R1). Dans son enseignement NEx (basket), Sam demeure tout aussi directif, mais ses injonctions sont effectuées de l'extérieur du milieu de l'élève. Sam ne joue pas avec les élèves, il s'attache plutôt à aménager progressivement leur milieu pour orienter leur activité décisionnelle. Par ailleurs, s'il démontre rarement, son échec lors d'une tentative de tir au panier le conduit à rappeler sa supériorité dans la pratique de l'activité : " d'accord, vous savez très bien que je suis pas très bon au shoot... donc... par contre en jeu, je risque de vous prendre, d'accord! » (B2).

\section{Tom : «Immerger»}

Tom est dans une logique d'immersion globale de la classe dans une culture commune transmise au travers de chaque activité enseignée (voir tableau 4). II engage les élèves directement dans des situations globales de pratique (des matchs en basket, des productions chorégraphiques en danse) ${ }^{17}$. La didactisation des activités sociales, particulièrement sous des formes scolaires classiques, est presque inexistante dans ses enseignements. D'autre part, Tom plonge les élèves dans le langage de l'activité enseignée, usant largement des signifiants propres aux champs lexicaux de celle-ci18 : "le shoot», «le tir en suspension », «le lancer franc », etc., en basket ; «le Trax », "le Pump », "le Sixstep », etc., en danse. Si cette logique d'immersion culturelle globale organise ses deux enseignements, elle prend une forme radicale dans son enseignement Ex (danse) et se spécifie par l'extériorité à cette immersion dans son enseignement NEx (basket).

En danse, Tom prend le rôle de l'expert en danse hip-hop à l'école de danse. L'immersion inaugurale se veut alors totale. Tom fait venir deux amis à lui, danseurs professionnels, tous les trois apprêtés dans les codes de la culture hip-hop (baggys, bonnets, etc.) et utilisant en permanence des références techniques ("les pass pass », " la new style », " le break », etc.) et artistiques ("Saïan Supa Crew », « le beat box », etc.). Après explicitation des fondements historiques du hip-hop (« il y a trente ans aux États-Unis, dans les quartiers noirs américains... " (D1), la classe devient alors une école de danse où trois experts, représentant l'instance Professeur (Sensevy, 2011), proposent aux élèves des pas de danse à enchaîner ensemble, comme des modèles à reproduire sur des rythmes hip-hop. Dans les séances suivantes, Tom enseigne seul en laissant les élèves construire des chorégraphies, très peu contraintes, où il s'agit simplement de 
respecter une durée, une occupation collective de l'espace, une formation de départ et de fin et de justifier leurs productions. Tom circule alors entre les groupes pour proposer, en les démontrant, des pas hip-hop à intégrer dans la chorégraphie. Sur un fond permanent de musique hip-hop, Tom danse entre les groupes, montrant corporellement ce qu'il est possible de faire en référence à la leçon inaugurale. Les élèves regardent alors les figures produites par Tom et s'attachent à les introduire dans leurs chorégraphies. En basket, si l'immersion s'effectue par la confrontation des élèves à des situations de jeu global et par l'utilisation d'un langage spécifique, cette immersion n'engage pas directement Tom. II demeure extérieur au milieu des élèves et s'adonne ponctuellement à quelques commentaires, principalement fondés sur des encouragements, par exemple lorsqu'un élève fait une passe décisive ("bien joué Simon "; B1), et quelques constats sur les actions des élèves, par exemple lorsqu'un d'entre eux échoue au tir au panier (" tu l'as pas tiré avec la planche »; B2).

La présentation de ces trois cas fait apparaître trois logiques didactiques qui spécifient alors le poids de l'expérience chez chaque sujet enseignant. L'analyse de leur discours peut maintenant permettre d'identifier les enjeux subjectifs sous-jacents à ces logiques didactiques et ainsi de comprendre davantage ce qui se joue ici pour chaque sujet.

\section{INTERPRÉTATIONS : LE POSITIONNEMENT DU SUJET DIDACTIQUE}

Nous poursuivons ici la construction des trois cas en confrontant chaque logique didactique dégagée au discours de chaque sujet et à nos cadres interprétatifs. L'effort de cohérence interne dans la construction de cas obéit ici « à l'éthique du bien dire, soit celle de trouver la formule qui traduit le mieux la position du sujet » (Terrisse \& Buznic-Bourgeacq, 2011).

\section{Trois sujets en position d'enseignant}

Charles - Fonction symbolique et appréhension : "avoir la place de mon prof"

Charles aborde de front, dans son discours aprèscoup, la situation difficile qu'a constituée la prise de responsabilité de la transmission du savoir : " je me sentais... impuissant... peu efficace... face aux responsabilités de la transmission » (AC). Quel que soit l'enseignement décrit, Charles souligne sa difficulté à intervenir directement « par le savoir » et explique alors la fonction des aménagements didactiques intensifs : " j'ai du mal à leur dire quelque chose, à leur donner des conseils différents qui vont leur permettre de plus réussir, donc à mon avis, ça passe par des situations " (AC). Faute de se sentir directement responsable des apprentissages des élèves, les situations peuvent jouer la fonction de l'enseignant à sa place. Dès lors, en tenant le contrat didactique, en le serrant autour d'une organisation didactique constante (" c'est toujours la même chose [...], comme ça on repartait sur quelque chose qui était déjà un peu fait »; $A C$ ), Charles peut reprendre une part de responsabilité par rapport à cette position qui lui semble s'échapper avant même qu'il soit entré en classe : " avant, j'appréhende vachement... la séance... j'appréhende... parce que bon, je sais pas trop où ça va aller, vu que je dépends de ce que eux font » (D1).

D'un « rapport craintif à la contingence " (BuznicBourgeacq, 2013), Charles s'instaure dans tous ses enseignements dans une logique d'appréhension : " je suis peut-être tellement dans l'organisation... je suis soucieux que ça se passe bien " (N1). Dès lors, le manque à savoir en danse et en basket ouvre encore un vide (« je peux pas me réfugier sur un truc que moi je sais »; D1), que la gestion serrée du contrat didactique peut alors sécuriser en constituant un " refuge » d'où Charles peut tenter d'enseigner. Dans cette situation, tout risque de symétrie est évité : « je montrais pas trop... parce que des fois, tu sais, tu tentes un panier, mais je le sentais pas... si je le rate, je ramasse ! ( $A C)$. Charles ne prend pas le risque de "ramasser " une blessure narcissique par une mise à l'épreuve ambigüe d'une position de maîtrise, qui témoignerait d'un décalage avec sa position supposée.

Si Charles désire être en position d'enseignant, il s'y réfère en extériorité, en fonction d'un ordre sur lequel il n'a pas encore prise, sans culpabilité, mais dans l'appréhension : " ma première année, tout ça, j'appréhendais... ça m'embêtait de ne pas être à la hauteur » (AC). Car cette position n'est pas, imaginairement, encore la sienne : "moi, ce que je trouve difficile, c'est quand même avoir la place de mon prof " (AC). En position d'élève dans le discours, Charles n'enseigne pas encore à la « hauteur » de son « prof ».

\section{Sam - Forme imaginaire et prestance : " c'était tellement... pour moi »}

Dans son discours après-coup, Sam revient sur les rôles distincts qu'il a endossés en rugby ("j'étais vraiment plus proche des élèves... et plus vraiment 
l'entraîneur très proche »; $A C$ ) et en basket (« j'ai balancé les situations et j'ai fait un peu l'animateur au niveau de la gestion humaine "; $A C)$. Une fois " entraîneur ", l'autre " animateur ", Sam ne se définit pas en premier lieu en tant qu'enseignant. S'il se distingue dans sa distance par rapport aux apprenants, d'un côté "proche des élèves ", de l'autre dans "la gestion humaine " au travers de " situations ", il n'en réfère pas à un manque à savoir ou à une faille dans la prise de responsabilité. C'est davantage une position plus ou moins défensive qui semble organiser cette distinction de lui-même : « en rugby j'étais décontracté, j'étais cool » $(A C)$; « en basket, je suis resté plus sur mes gardes " (AC). Pas d'appréhension chez Sam à défaut d'un savoir supposé, mais plutôt la protection d'une image plus ou moins idéalisée.

Le maintien de cette " place de premier » (Terrisse, 1999), appelant moins à la responsabilité qu'à la rivalité ou à la séduction, nous semble être révélateur de l'ambivalence qui organise les pratiques de Sam. Les nombreux retours sur sa propre performance corporelle en rugby et la réaction défensive adoptée en basket dès son échec dans la mise à l'épreuve délicate de sa place de " maître » témoignent de ce positionnement. Sam enseigne dans une relation duale avec les élèves, en position de pratiquant, au risque de la symétrie: " je reprends la situation en me mettant moi à leur place » (R2). Il laisse s'effacer la position symbolique d'où il peut incarner sa fonction supposée pour agir parmi ses semblables, pratiquants de rugby. Mais enseignant en place de pratiquant, l'assomption d'une image satisfaisante narcissiquement ne peut se faire de manière identique. Dans une logique de prestance, Sam peut enseigner aisément " pour lui » en rugby : " en rugby c'était tellement... pour moi... je faisais les choses tellement facilement, que c'était... ça passait facilement pour moi... ". Mais, dans la rivalité, il y perd sa place idéale en basket : " là en basket il y avait des élèves meilleurs que moi...» (AC).

En deçà du nouage entre les enjeux narcissiques et la fonction symbolique de l'enseignant qui peut le conduire à s'assurer d'une image de maîtrise pour soutenir sa fonction supposée, Sam s'attache à assumer cette image sans détour symbolique. II enseigne moins dans une tentative d'alignement avec sa fonction que dans une affirmation de son image ou un évitement direct d'une blessure narcissique. La relation de prestance ou de rivalité qui lie Sam à ses élèves témoigne de l'image de maîtrise idéale qu'il reflète sur eux et dont le maintien organise ses pratiques d'enseignement.
Tom - Impossible et crédibilité : "j'étais beaucoup plus le grand frère »

L'importance des transferts des élèves sur l'enseignant est au cœur du discours de Tom : "l'élève il sent que t'es compétent dans une matière " (AC). Cette compétence est pour lui gage de sauvegarde de l'enseignant: "si t'es pas capable de montrer ce que c'est qu'un double pas, t'es fichu " (AC). Tom explique alors les soubassements des différences d'enseignement en basket et en danse : « je me suis beaucoup plus impliqué parce que je me sentais beaucoup plus crédible à leurs yeux en hip-hop qu'en basket » (AC). Le sentiment de compétence projetée sur les élèves s'inscrit ici dans un jeu de miroir et se renverse en sentiment de crédibilité de son propre côté.

Ce souci de crédibilité est en effet déterminant. II conduit par exemple Tom à une forme d'enseignement globale du basket, simplement assortie de quelques commentaires en extériorité : « je suis resté quand même assez global sur le basket, parce que moi j'aime pas non plus me mentir à moi-même " (AC). Pas de tentative d'illusion, d'appréhension, ou de position défensive pour Tom à défaut d'expérience personnelle, plutôt un décalage par rapport à sa fonction et aux formes scolaires symboliquement attendues, par lequel, à défaut d'assumer une position d'enseignant, il ne se ment pas à lui-même. Mais ce premier positionnement ne se fait pas sans une certaine forme de regret, éclairant plus encore la logique de Tom : « en basket, c'était quand même un cycle beaucoup plus froid » (AC). Ce n'est pas l'assomption d'une position symbolique, difficile à tenir, qui organise Tom, mais bien le manque d'un rapport " plus humain » avec «plus d'affect» (AC). Chose qu'il parvient à trouver dans son engagement proximal et corporel en danse, au travers d'une position subversive de la forme scolaire : "dans le hip-hop, j'étais beaucoup plus le grand frère » $(A C)$.

Le manque d'affect et de rapport humain ouvert par l'assujettissement à une position symbolique d'enseignant ramène ainsi Tom vers sa place dans un autre ordre, non proprement didactique. Car l'assujettissement conduit à effacer l'image à laquelle Tom s'identifie idéalement pour demeurer crédible à lui-même : " moi, ils m'imaginent pas en danseur professionnel, je veux dire, tu vois... enseignant, ils ne m'imaginent pas » (D1). Tom enseigne à partir de ce qu'il est, un " grand frère ", un « danseur professionnel » et il lui est impossible à supporter de ne pas être imaginé ainsi. 


\section{Conclusion : l'expérience du sujet, la position de l'enseignant}

Les cas présentés mettent au jour les trajectoires de trois sujets dans leur construction singulière d'une position d'enseignant débutant. L'analyse didactique a permis d'identifier dans leurs pratiques des logiques didactiques singulières, tant du point de vue épistémologique des contenus - des pratiques, des automatismes, une culture - que du point de vue interactionnel des processus didactiques - une gestion intensive du contrat didactique, une répétition, une immersion globale. L'analyse didactique clinique a permis de dégager dans le discours de chaque enseignant des problématiques propres au sujet didactique. L'articulation entre une fonction supposée par rapport à laquelle chacun s'aligne ou se décale, les enjeux narcissiques immanents à l'assomption d'une image reflétée sur l'élève et le manque à être qui émerge de cette réduction du sujet à une fonction et une forme, constitue alors un système d'interprétation des logiques reconstruites en amont.

Cette analyse a conduit à repenser la dialectique " $\mathrm{Ex} / \mathrm{NEx}$ » comme problématique singulière à chaque sujet enseignant. Si des tendances permettent clairement d'identifier une influence générique de l'expérience de pratiquant de l'enseignant sur son activité didactique, on a pu voir combien la manière dont chaque sujet se situe singulièrement face à une position d'enseignant détermine d'autant plus l'intensité et la forme de cette influence. Ainsi, l'alignement de Charles à une fonction symbolique supposée s'actualise dans une position singulière - d'appréhension - qui donne forme à une activité didactique semblable qu'il ait ou non une expérience personnelle. Quelle que soit son expérience, Charles témoigne d'une absence de prise sur la responsabilité de la transmission et ainsi d'un décalage permanent à sa fonction supposée qu'il tente de réduire par l'intermédiaire d'une organisation didactique intensive. À l'inverse, les détours de Sam par une position imaginaire - de prestance - et de Tom par une position subversive - de crédibilité - conduisent à des activités didactiques plus contrastées. Le maintien d'une image de maîtrise idéale conduit Sam à rester sur ses gardes dans un climat de rivalité ou bien à affirmer son excellence corporelle parmi les élèves. Le souci de crédibilité de lui-même qui organise Tom l'amène à animer de loin une forme globale de pratique non maîtrisée ou bien à enseigner au plus près des élèves en position de "grand frère " une pratique dans laquelle il s'identifie d'abord comme «professionnel ».

Chacun se situe ainsi singulièrement comme sujet/ enseignant, à partir de son expérience personnelle et face à une position qui le définit sans être prédéfinie, en tant qu' " entraîneur » ou " animateur ", " danseur professionnel » et " grand-frère ", " à la place des élèves ", dans le manque ouvert par "la place de mon prof ". Mais il s'agit bien d'un manque consistant, une distance du sujet à sa position d'enseignant, comme figure du désir d'enseigner, dont la prise en compte peut permettre au chercheur comme au formateur d'écouter d'où le sujet transmet.

Pablo Buznic-Bourgeacq pablo.buznic-bourgeacq@unicaen.fr Université de Caen Basse-Normandie, CERSE, Université Toulouse 2-Le Mirail, EDIC, UMR EFTS

André Terrisse andre.terrisse@wanadoo.fr Université Toulouse 2-Le Mirail, EDIC, UMR EFTS 
1 Issus de la didactique des mathématiques, plusieurs outils élaborés au travers de ces travaux ont montré leur opérationnalité et leur heuristique dans d'autres champs disciplinaires (Salin, Clanche \& Sarrazy, 2005 ; Merri, 2007), particulièrement en didactique de l'EPS (Loquet, Amade-Escot \& Marsenach, 2005 ; Le Bas, 2007 ; Récopé, 1996).

2 Ces indicateurs empiriques sont nombreux et émergent d'un cadre conceptuel systémique. Ils s'organisent en plusieurs éléments constitutifs d'un cadre d'analyse des pratiques d'enseignement. L'objet de cet article n'étant pas l'explicitation de ce cadre d'analyse didactique, le lecteur intéressé pourra se référer à Buznic-Bourgeacq, Terrisse \& Margnes (2010) ou, pour une version complète, à Buznic-Bourgeacq (2009). Nous en présentons toutefois plusieurs aspects dans la méthodologie et les résultats exposés dans cet article.

3 II faut entendre ici le sujet dans sa globalité, car le registre de l'imaginaire est plus spécifiquement le registre privilégié du Moi. Or, « le Moi n'est toujours que la moitié du sujet » (Lacan, 1966, p. 346). C'est son inscription dans le symbolique qui le fait sujet, l'assujettit à l'ordre symbolique qui le précède et le détermine.

4 Une caméra en plan large focalisée en permanence sur les enseignants a permis d'enregistrer au final $28 \mathrm{~h}$ de séances d'EPS. Les enseignants étant équipés d'un micro-cravate, nous avons pu enregistrer l'ensemble de leurs interactions verbales.

5 Il s'agit ici de l'ensemble des procédures didactiques au travers desquelles l'enseignant "donne à voir " du savoir (en dehors des interactions verbales) pour guider l'élève. II peut démontrer corporellement le savoir, il peut manipuler le corps de l'élève, il peut faire un schéma au tableau.

6 L'entretien d'après-coup, outil méthodologique privilégié en didactique clinique, se caractérise par le temps conséquent qui le sépare de toutes les premières étapes de la recherche. Postulant que le point de vue du sujet sur sa propre pratique, et non pas de l'observateur de sa propre pratique, s'établit au travers d'un " remaniement » (Freud, [1933] 1995), il nécessite un certain éloignement du vif des pratiques.

7 Diversité des signifiants participant à la conceptualisation de l'activité du pratiquant dans l'ASPA utilisés par les enseignants en moyenne sur trois séances : $E x=104,8$; NEx $=66,2$. Diversité des signifiants du champ lexical de l'APSA utilisés par les enseignants en moyenne sur trois séances : Ex $=16,2$; NEx $=$ 6,2 .
8 Nombre moyen d'analogies internes (en référence à des éléments ayant déjà émergé en classe) explicitées par les enseignant sur trois séances: $\mathrm{Ex}=13,8 ; \mathrm{NEx}=5,4$. Nombre moyen d'analogies externes (comparaisons et métaphores en référence à des éléments extérieurs à la classe) explicitées par les enseignants sur trois séances: $\mathrm{Ex}=13,8 ; \mathrm{NEx}=6,4$.

9 Diversité des invariants mis en jeu par les enseignants en moyenne sur trois séances: $\mathrm{Ex}=32,8 ; \mathrm{NEx}=19,6$.

10 Pourcentage moyen de situations globales, non décomplexifiées structurellement par rapport à la pratique sociale de référence, constituant la diversité des situations d'apprentissage mises en œuvre par les enseignants sur trois séances : $E x=29,2 \%$; NEx $=10,8 \%$.

11 Nombre moyen de démonstrations corporelles effectuées par les enseignants sur trois séances : $\mathrm{Ex}=136$; NEx = 25,4

12 Pourcentage moyen d'interventions des enseignants centrées sur l'articulation entre la situation singulière dans laquelle se trouve l'élève et des éléments de savoir génériques sur trois séances : $E x=40,8 \% ; N E x=21,8 \%$.

13 Les extraits de séances et d'entretiens sont référés par un code. Ils sont codés par la première lettre de l'APSA enseignée, suivie du numéro de la séance dont il s'agit (par exemple, deuxième séance de rugby : R2). Pour les entretiens d'après-coup, on notera AC.

14 La diversité et la redondance des objets de savoir mis en jeu, des signifiants de l'activité enseignée, ainsi que le nombre de démonstrations corporelles mises en œuvre par l'enseignant sont minimaux dans tous les enseignements de Charles.

15 Le nombre de règles d'actions mises en œuvre par l'enseignant est maximal dans tous les enseignements de Sam.

16 Le nombre de démonstrations corporelles mises en œuvre par l'enseignant est maximal dans l'enseignement Ex de Sam.

17 La proportion de situations " globales" (sans variable didactique transformant la forme sociale de pratique de l'activité) est maximale dans tous les enseignements de Tom.

18 La diversité des signifiants appartenant au champ lexical de l'activité enseignée mis en jeu par l'enseignant est maximale dans tous les enseignements de Tom. 


\section{BIBLIOGRAPHIE}

ALTET M. (1996). « Les compétences de l'enseignant professionnel : entre savoirs, schèmes d'action et adaptation, le savoir analyser ". In L. Paquay, M. Altet, E. Charlier \& P. Perrenoud (dir.), Former des enseignants professionnels. Quelles stratégies ? Quelles compétences? Bruxelles: De Boeck.

ARNAUD P. (1986). « Objet culturel, objet technique, objet didactique ». Revue STAPS, vol. 13, p. 43-55.

ASSOUN P.-L. (2003). Lacan. Paris : PUF.

BEN JOMAA H. (2011). «De l'expertise à l'enseignement : analyse comparative du rapport au savoir de deux enseignants d'EPS de spécialités sportives différentes ". Éducation et didactique, vol. 5, n॰ 3, p. 61-80.

BLANCHARD-LAVILLE C. (2001). Les enseignants, entre plaisir et souffrance. Paris : PUF.

BROUSSEAU G. (1998). Théorie des situations didactiques. Grenoble : La Pensée sauvage.

BUZNIC-BOURGEACQ P. (2009). La transmission du savoir expérientiel. Études de cas et analyses comparative en didactique clinique de l'EPS. Thèse de doctorat, didactique des disciplines scientifiques et technologiques, université Toulouse 3.

BUZNIC-BOURGEACQ P. (2013). « La contingence de I'enseignement ou la mise à l'épreuve du sujet supposé savoir ». In M.-F. Carnus \& A. Terrisse (dir.), Didactique clinique de l'EPS. Le sujet enseignant en question. Paris : Éd. Revue EPS.

BUZNIC-BOURGEACQ P., TERRISSE A. \& LESTEL G. (2008). «Expérience personnelle et expérience professionnelle dans l'enseignement de l'EPS : deux études de cas contrastés en didactique clinique ". Éducation et didactique, vol. 2, no 3, p. 77-96.

BUZNIC-BOURGEACQ P., TERRISSE A. \& MARGNES E. (2007). « L'expérience personnelle du professeur d'EPS, approche clinique et implications didactiques: une étude de cas d'une enseignante débutante ". eJRIEPS, no 11 , p. 20-39.

BUZNIC-BOURGEACQ P., TERRISSE A. \& MARGNES E. (2010). « La transmission du savoir expérientiel en EPS. Études de cas et analyses comparatives en didactique clinique ». eJRIEPS, no 20, p. 26-47.

CARNUS M.-F. (2010). « La construction de la professionnalité enseignante à travers un dispositif interdisciplinaire de collège ". Revue française de pédagogie, no 173, p. $19-40$.

CARNUS M.-F. \& TERRISSE A. (2013). Didactique clinique de l'EPS. Le sujet enseignant en questions. Paris : Éd. Revue EPS.

CHEMAMA R. (2009). Dictionnaire de la psychanalyse. Paris : Larousse.

CHEVALLARD Y. (1991). La transposition didactique, du savoir savant au savoir enseigné. Grenoble : La Pensée sauvage.

FILLOUX J. (1996). Du contrat pédagogique. Le discours inconscient de l'école. Paris : L'Harmattan.

FREUD S. ([1933] 1995). « Nouvelle suite de leçons d'introduction à la psychanalyse. XXXIVe leçon : éclaircissements, applications, orientations ». In S. Freud, CEuvres complètes : psychanalyse. Paris : PUF.
LACAN J. (1966). Écrits. Paris : Éd. du Seuil.

LACAN J. (1974). «RSI ». Le Séminaire, livre XXII. Inédit.

LACAN J. (2001). Autres écrits. Paris : Éd. du Seuil.

LE BAS A. (2007). «Didactique professionnelle, formation des enseignants et problématisation ». Recherches en éducation, no 3, p. 109-124.

LOQUET M., GARNIER A. \& AMADE-ESCOT C. (2002). "Transmission des savoirs en activités physiques, sportives et artistiques dans des institutions différentes : enseignement scolaire, entraînement sportif, transmission chorégraphique ». Revue française de pédagogie, no 141, p. 99-109.

LOQUET M., AMADE-ESCOT C. \& MARSENACH J. (2005). "Théorie des situations didactiques en éducation physique. Pourquoi ? Comment ? Quelle transposition ? ". In M.-H. Salin, P. Clanché \& B. Sarrazy (dir.), Sur la théorie des situations didactiques. Grenoble : La Pensée sauvage.

MERCIER A., SCHUBAUER-LEONI M.-L. \& SENSEVY G. (2002). "Vers une didactique comparée ». Revue française de pédagogie, no 141, p. 5-16.

MERRI M. (2007). Activité humaine et conceptualisation. Toulouse : Presses universitaires du Mirail.

PAQUAY L. (1994). « Vers un référentiel de compétences professionnelles de l'enseignant ? ". Recherche et formation, no 15, p. 7-35.

PASSERON J.-C. \& REVEL C. (2005). Penser par cas. Paris : Éd. de l'EHESS.

PHILIPPOT T. (2008). La professionnalité des enseignants de l'école primaire. Savoirs et pratiques. Thèse de doctorat, sciences de l'éducation, université de Reims Champagne-Ardenne.

PUJADE-RENAUD C. (1983). Le corps de l'enseignant dans la classe. Paris : L'Harmattan.

RÉCOPÉ M. (1996). Statut et fonctions du schéma de duel dans l'organisation de l'action motrice d'opposition (le cas du volley-ball). Thèse de doctorat, psychologie, université Paris Descartes-Paris 5.

REVAULT D'ALLONNES C. (1989). La démarche clinique en sciences humaines. Paris : Dunod.

SALIN M.-H. (2002). " Les pratiques ostensives d'un enseignement des mathématiques comme objet d'analyse du travail du professeur ". In P. Venturini, C. AmadeEscot \& A. Terrisse (dir.), Études des pratiques effectives: l'approche des didactiques. Grenoble : La Pensée sauvage.

SALIN M.-H., CLANCHE P. \& SARRAZY B. (2005). Sur la théorie des situations didactiques. Grenoble : La Pensée sauvage.

SENSEVY G. (2011). Le sens du savoir. Bruxelles : De Boeck.

SHULMAN L.-S. (1986). «Those Who Understand: Knowledge Growth in Teaching ". Educational Researcher, vol. 15 , no 2, p. 4-14.

TERRISSE A. (1994). La question du savoir dans la didactique des activités physiques et sportives : essai de formalisation. Habilitation à diriger les recherches, didac- 
tique des activités physiques et sportives, université Toulouse 3-Paul Sabatier.

TERRISSE A. (1999). " La question du rapport au savoir dans le processus d'enseignement-apprentissage : le point de vue de la clinique ". Carrefours de l'éducation, no 7, p. 62-87.

TERRISSE A. (2000). "Épistémologie de la recherche clinique en sports de combat ». In A. Terrisse (dir.), Recherches en sports de combat et en arts martiaux: état des lieux. Paris: Éd. Revue EPS.

TERRISSE A. (2001). « La référence dans l'enseignement de l'éducation physique et sportive ". In A. Terrisse (dir.), Didactique des disciplines. Les références au savoir. Bruxelles : De Boeck.

TERRISSE A. (2007). «L'après-coup en didactique de I'EPS : procédures et effets ». In D. Lahanier-Reuter \& E. Roditi (2007), Les méthodes de recherche en didactique (2). Questions de temporalité. Lille : Presses universitaires du Septentrion.

TERRISSE A. (2008). " Le sujet en didactique clinique de I'EPS : conditions et conséquences pour la recherche ». eJRIEPS, no 15, p. 179-197.

TERRISSE A. (2009). « La didactique clinique en EPS. Origine, cadre théorique et recherches empiriques ". In A. Terrisse \& M.-F. Carnus (dir.), Didactique de l'éducation physique et sportive. Quels enjeux de savoir? Bruxelles : De Boeck.
TERRISSE A. \& BUZNIC-BOURGEACQ P. (2011). « La validation scientifique des recherches en didactique clinique : la construction de cas ". Estilos da clinica, vol. 15, no 2, p. 346-361.

TERRISSE A. \& CARNUS M.-F. (2009). Didactique de l'éducation physique et sportive. Quels enjeux de savoir? Bruxelles: De Boeck.

TERRISSE A., CARNUS M.-F. \& SAUVEGRAIN J.-M. (2002). "Le rapport au savoir : point de vue psychanalytique et recherches en didactique de l'EPS ". Actes des 3es journées franco-québécoises de didactique : rapport au savoir et didactiques. Paris : Sorbonne.

TERRISSE A. \& LABRIDY F. (1991). «Imaginaire, Réel, Symbolique et acte sportif ". Dossier EPS, no 10, p. 405412.

TOUBOUL A., CARNUS M.-F. \& TERRISSE A. (2012). « Les effets de l'expérience et de l'expertise dans les pratiques d'enseignement en éducation physique et sportive : analyse de deux profils asymétriques en savate boxe française ". Recherches en didactique, no 12, p. 47-65.

VERGNAUD G. (1990). "La théorie des champs conceptuels ". Recherches en didactique des mathématiques, vol. 10, no 2-3, p. 133-170.

VERGNAUD G. (2002). «Forme opératoire et forme prédicative de la connaissance. Conférence introductive ". Actes de l'université d'été du CIFEN, p. 10-18. 